\title{
ARCHÉOLOGIE ET MISE EN VALEUR DE L'ÎLE SAINT-BARNABÉ (RIMOUSKI, QUÉBEC) : DE LA MATÉRIALISATION D'UN MYTHE À LA PERFORMANCE ARCHÉOLOGIQUE
}

\author{
Arqueologia e valorização da Ilha Saint-Barnabé \\ (Rimouski, Québec): da materialização de um mito à \\ performance arqueológica \\ Manon Savard* \\ Nicolas Beaudry**
}

\begin{abstract}
This article offers reflections on the experience of a long-term archaeological investigation in an academic context and is interested in the evolution of the objectives, expectations, and interests of its various actors. The project combined research, education, and development, seeking a balance among these three dimensions of the project that fed each other. This balance allowed a place for the interests of each of the actors: those of the university professor-researchers and students, those of the local municipality and its tourism promotion agency, and those of the general public. Over time, the education mission of the project has taken on a more important role and the development has gradually taken the form of an archaeological performance. The authors question the long-term viability of this type of development.
\end{abstract}

* Professeure, Département de biologie, chimie et géographie. Université du Québec à Rimouski. 300, allée des Ursulines, C.P. 3300, succ. A. Rimouski (Québec) Canada. G5L 3A1. Courriel : manon_savard@uqar.ca

Professeur, Département des lettres et humanités. Université du Québec à Rimouski. 300, allée des Ursulines, C.P. 3300, succ. A. Rimouski (Québec) Canada. G5L 3A1

1 Manon Savard et Nicolas Beaudry sont des co fondateurs du Laboratoire d'archéologie et de patrimoine et co responsables de l'École de fouilles archéologiques, à l’Université du Québec à Rimouski, Québec. 
Keywords: Public archaeology; Archaeological pedagogy; Development; Archaeological performance

\section{RÉSUMÉ}

Cet article propose un retour sur une expérience d'intervention archéologique de longue durée en contexte universitaire et s'intéresse à l'évolution des objectifs, des attentes et des intérêts de ses différents acteurs. L'intervention conciliait recherche, enseignement et mise en valeur, cherchant un équilibre entre ces trois dimensions qui se nourrissaient mutuellement. Cet équilibre permettait de répondre aux intérêts de chacun des acteurs : ceux de l'université, les professeurs-chercheurs et des étudiants, ceux de la municipalité et de son organisme de promotion touristique, ceux du public en général. Au fil du temps, l'enseignement a pris une dimension de plus en plus affirmée et la mise en valeur a graduellement pris la forme d'une performance archéologique. Les auteurs s'interrogent sur la viabilité à long terme d'une telle forme de mise en valeur.

Mots-clés: archéologie publique; enseignement de l'archéologie; mise en valeur; performance archéologique

\section{RESUMO}

Este artigo estuda a evolução dos objetivos, das expectativas e dos interesses dos diferentes atores de uma experiência de intervenção arqueológica de longo prazo realizada no contexto universitário. A intervenção conciliava pesquisa, ensino e valorização, buscando equilibrar estas três dimensões que alimentavam reciprocamente. Este equilíbrio permitiu responder aos interesses de cada um dos atores: os da universidade, dos professores-pesquisadores e dos estudantes, os da municipalidade e do seu órgão de promoção turística, e os do publico em geral. Ao longo do tempo, o ensino assumiu uma importância cada vez maior e a valorização progressivamente assumiu a forma de uma performance arqueológica. Os autores questionam-se sobre a viabilidade à longo prazo deste tipo de valorização.

Palavras-chave: arqueologia publica; ensino da arqueologia; valorização; performance arqueológica 


\section{Introduction}

De 2009 à 2014, des professeurs-chercheurs et des étudiants de l'Université du Québec à Rimouski (UQAR) ont mené chaque été des opérations archéologiques sur l'île Saint-Barnabé, au large de Rimouski (Québec, Canada), dans l'estuaire du Saint-Laurent ${ }^{2}$ (figure 1). Les objectifs, le caractère et le déploiement des activités archéologiques ont connu des changements profonds au cours de ces six saisons. Alors que le projet devait initialement contribuer à une mise en valeur éclairée du site et de l'île, cette mise en valeur a graduellement pris la forme d'une performance archéologique. Cet article présente le projet et son contexte; il relate l'évolution du projet et des intérêts de ces principaux acteurs, puis propose quelques réflexions sur la performance archéologique comme forme de mise en valeur.

Figure 1

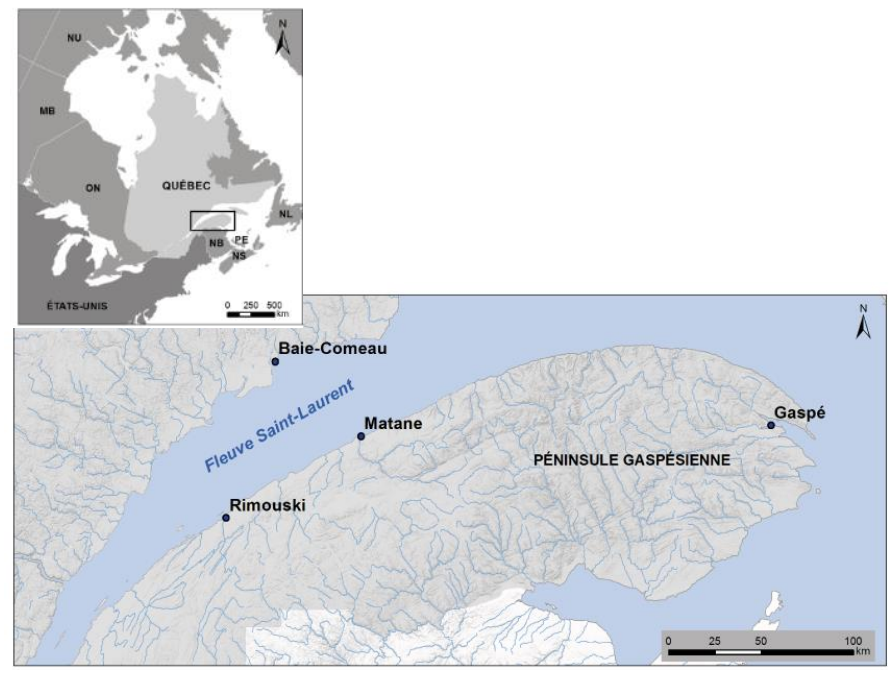

Localisation de Rimouski (Marie-Andrée Roy, UQAR)

2 Ces travaux ont été financés par la Ville de Rimouski et, de 2012 à 2014, par le projet PATER (UQAR-Cégep de Rimouski, Québec), lui-même financé par le ministère Québécois de l'Éducation, du Loisir et du Sport. Il a également bénéficié de contributions de l'UQAR et de la Fondation Héritage Canada. 


\section{Rimouski et l'île Saint-Barnabé}

L'ile Saint-Barnabé est située à 3 kilomètres au large de Rimouski, la capitale administrative de la région québécoise du BasSaint-Laurent, sur la rive sud de l'estuaire du Saint-Laurent ${ }^{3}$. Il s'agit d'une barre appalachienne formée de schiste qui se présente comme une longue et étroite bande de terre (6 kilomètres de longueur par moins de 0,5 kilomètres de largeur) qui ferme la baie de Rimouski. Sa topographie présente peu de variations, à l'exception d'une crête rocheuse dont l'altitude dépasse à peine 10 mètres; son sommet, appelé la Petite Montagne, atteint 17 mètres. La ville elle-même est établie sur des paléo-terrasses marines qui se déploient comme les gradins d'un immense théâtre naturel dont l'île Saint-Barnabé serait la scène (figure 2). L'île Saint-Barnabé domine l'imaginaire des Rimouskois autant qu'elle domine leur paysage. Elle n'est qu'à faible distance de la côte et peut facilement être atteinte en chaloupe ou en kayak quand la mer est calme, voire à pied lorsque la marée est très basse, mais les conditions maritimes peuvent changer rapidement dans l'estuaire et l'île peut alors être beaucoup plus lointaine qu'elle ne le semble. Son accès a longtemps été restreint par ses propriétaires et leurs gardiens.

L'île est la scène de récits plus ou moins légendaires de naufrages, de baleines échouées, de vigies à l'affût de navires anglais et de sous-marins allemands, de contrebande d'alcool, de parties de chasse des élites rimouskoises, tous encore très vivants dans l'histoire écrite et orale. Des cultivateurs aux contrebandiers et aux bûcherons, les insulaires habitent encore la mémoire collective des Rimouskois ${ }^{4}$.

3 La Ville de Rimouski compte 49197 habitants en 2017 (Ville de Rimouski, données statistiques de

2017 ,

http://www.ville.rimouski.qc.ca/fr/decouvrezRimouski/nav/portrait.html?iddoc=140513).

4 MASSICOTTE, Marie-Andrée, et LECHASSEUR, Antonio. De verdure et de rêves, l'île Saint-Barnabé. Rimouski: Société Joseph-Gauvreau pour le patrimoine, 1988; FORTIN, JeanCharles, et LECHASSEUR, Antonio. Histoire du Bas-Saint-Laurent. Québec: Institut québécois de recherche sur la culture, 1993; MASSICOTTE, Marie-Andrée. Une île au large de la ville. Revue d'histoire du Bas-Saint-Laurent, n. 19 (2 ), 1996, p. 57-62; CHASSÉ, Béatrice. Rimouski et son île. Les seigneurs Lepage et l'île Saint-Barnabé. Rimouski: Société d'histoire du Bas-Saint-Laurent / Groupe de recherche interdisciplinaire sur le développement régional de l'Est du Québec, 2003; 
Figure 2

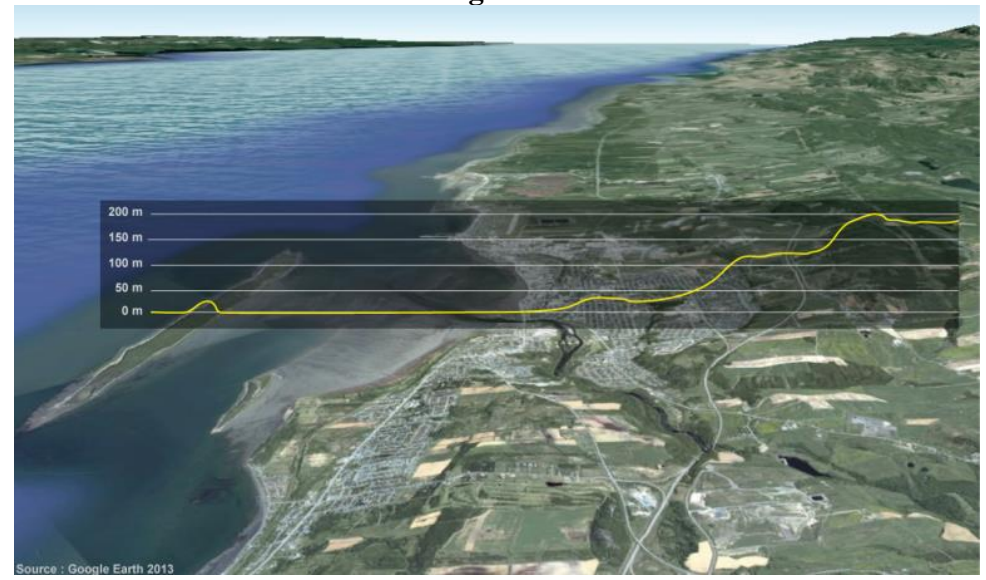

Topographie de l'île Saint-Barnabé et de Rimouski (Marie-Andrée-Roy, UQAR)

La plus grande partie de l'île appartient aujourd'hui à la Ville de Rimouski, qui en a fait un parc. Tourisme Rimouski assure un service de navette par bateau pneumatique pendant la saison touristique, c'est-à-dire de la fin juin à début septembre ; elle occupe aujourd'hui une place enviable dans l'offre touristique de la région de Rimouski.

\section{Toussaint Cartier, l'ermite de l'île Saint-Barnabé}

L'un de ces insulaires occupe une place particulière dans l'imaginaire des Rimouskois : le premier habitant connu de l'île, Toussaint Cartier, dit l'ermite, qui y a vécu en solitaire d'environ 
1728 jusqu'à sa mort en $1767^{5}$. Il suscitait déjà la curiosité de son vivant, comme en témoigne une note dans le journal du commandant des troupes françaises en Amérique du Nord, le marquis de Montcalm, alors qu'il passe au large de l'île ${ }^{6}$. Moins de deux ans après sa mort, il apparaît dans un roman de l'anglaise Frances Brooke sous la forme d'un veuf qui se serait retiré du monde pour pleurer sa bien-aimée ${ }^{7}$. Près d'un siècle plus tard, l'archevêque de Québec, $\mathrm{M}^{\mathrm{gr}}$ Joseph Signay, publie le portrait d'un dévot qui aurait consacré son existence à la contemplation et à la prière ${ }^{8}$; ce personnage de chrétien exemplaire a été largement instrumentalisé par l'Église de Rimouski, ce qui explique des regains d'intérêt lors de l'érection canonique de la paroisse de Rimouski en 1827 et, de nouveau, à la veille de l'érection du diocèse de Rimouski en 1867, 100 ans après la mort de l'ermite. Depuis, les personnages de Brooke, de Signay et d'autres auteurs ${ }^{9}$ se sont croisés, contribuant à construire un personnage légendaire, ambigu et changeant, auquel les Rimouskois sont restés très attachés et qui continue de les intriguer. D'autres

5 MIMEAULT, Mario. La légende Toussaint Cartier: critique des sources. Revue d'histoire du Bas-Saint-Laurent, n. 14(1), 1990, p. 23-30; GOSSELIN, Sylvain. Le territoire et ses premiers occupants, In: Paul Larocque (éd.). Rimouski depuis ses origines. Rimouski: Société d'histoire du Bas-Saint-Laurent / Société de généalogie et d'histoire de Rimouski / GRIDEQ, 2006, p. 19-91; SCALLON-CHOUINARD, Pascal. Un homme à la marge. Toussaint Cartier, ermite de l'île Saint-Barnabé. Revue d'histoire de l'Université de Sherbrooke, n. 3 (1), 2012, < www.rhus.association.usherbrooke.ca/wp-content/articles/312.pdf . ; LA CHARITÉ, Claude. L'érémitisme en Nouvelle-France et la vocation singulière de Toussaint Cartier au XVIIIe siècle. Études d'histoire religieuse, n. 79(2), 2013. p. 5-19.

6 "L'île Saint-Barnabé [...] est habitée par un gentilhomme breton des environs de Morlaix, qui par singularité ou dévotion y mène la vie d'un ermite, et se sauve même dans les bois, si on cherche à l'aborder lorsque les bâtiments y mouillent": MONTCALM, Louis-Joseph de. Journal du Marquis de Montcalm durant ses campagnes en Canada de 1756 à 1759, éd. par H.R. CASGRAIN, Québec: Imprimerie L.-J. Demers et frères, 1895.

7 BROOKE, Frances. The History of Emily Montague. Londres: J. Dodsle, 1769. V. aussi MIGNEAULT, Yvon. Que devons-nous à Frances Brooke, 1724-1789, au sujet de Toussaint Cartier, l'ermite de l'île Saint-Barnabé, 1707-1767? Revue d'histoire du Bas Saint-Laurent, n. 13(1), 1988, p. 3-11.

8 SIGNAY, Joseph, Notice sur le nommé Toussaint Cartier surnommé l'ermite de l'île Saint-Barnabé, mort et enterré à Rimouski en 1767. L'Abeille, n. 10 (22), 31 mai 1862.

9 Le personnage du dévot a reçu sa forme la plus achevée et la plus influente sous la plume de Joseph-Charles Taché, écrivain polygraphe et homme politique rimouskois . voir TACHÉ, Joseph-Charles. L'île Saint-Barnabé dans le comté et dans la paroisse de Rimouski. Les soirées canadiennes, n. 5, 1865, p. 341-365; TACHÉ, Joseph-Charles. Les Sablons ('île de Sable) et l'île Saint-Barnabé. Montréal : Librairie Saint-Joseph, Cadieux \& Derome, 1885, p. 135-154. 
poètes et des écrivains ${ }^{10}$ l'ont évoqué depuis Frances Brooke; il réapparaît régulièrement dans la presse régionale ${ }^{11}$; des historiens et des érudits poursuivent leur enquête sur le personnage ${ }^{12}$ et des artistes s'en inspirent ${ }^{13}$. Le personnage de l'ermite a même donné son nom à une discothèque rimouskoise ${ }^{14}$, qui n'a cependant pas vécu très longtemps.

\section{Les objectifs de l'intervention archéologique}

Les premières interventions ont été initiées à l'invitation de l'organisme gestionnaire de l'île, Tourisme Rimouski, qui souhaitait enrichir l'offre touristique régionale par l'interprétation historique de l'île qui appartient presque entièrement à la Ville de Rimouski et qui accueille randonneurs et campeurs pendant la saison estivale. On attendait des archéologues qu'ils valident un discours historique centré sur le premier habitant connu de l'île au XVIII ${ }^{\mathrm{e}}$ siècle, l'ermite Toussaint-Cartier, et qu'ils l'illustrent en fournissant des artefacts qui seraient exposés dans un aménagement prévu sur le site où il aurait vécu. Les travaux ont plutôt livré la matière d'un récit plus riche, qui dépasse la seule personne de l'ermite et qui a ouvert des perspectives plus larges de mise en valeur, de la préhistoire jusqu'au $\mathrm{XX}^{\mathrm{e}}$ siècle. L'implication d'étudiants dans les travaux archéologiques se faisant plus soutenue, le caractère pédagogique des interventions s'est développé jusqu'à leur donner la forme d'une école de fouilles. Le

10 LA CHARITÉ, op. cit.

11 SCALLON-CHOUINARD, op. cit.

12 On mentionnera en particulier Claude La Charité, professeur à l'UQAR et titulaire de la Chaire de recherche du Canada en histoire littéraire et patrimoine imprimé. Ses recherches donnent lieu à une série d'articles dans Le Mouton NOIR (Rimouski) : voir LA CHARITÉ, op. cit., p. 6 , n. 2 .

13 En 2013, Guillaume Boudrias-Plouffe présentait au Centre d'art Caravansérail de Rimouski une installation intitulée Coudon! Yé pas sorti du bois !, inspirée de la vie de Toussaint Cartier: voir MATHIEU, S., Caravansérail : incursion dans l'univers de Toussaint Cartier. L'Avantage, Rimouski, 10 avril 2013, p. 20.

Rimouski, 22 mars 2009.

14 MICHAUD, Pierre. Le Chiff cède sa place au Toussaint Cartier. Le Progrès Écho, 
site étant ouvert au public, les visiteurs ont été intégrés à la fois dans la démarche d'enseignement et dans la démarche de recherche. Leurs intérêts et ceux de Tourisme Rimouski s'ouvraient alors à d'autres occupations que celle de l'ermite et à d'autres personnages, jusqu'aux occupations récentes et jusqu'aux archéologues eux-mêmes. Ces derniers comptent désormais parmi les insulaires et leur performance archéologique contribue à l'animation de l'île.

\section{L'archéologie et la mise en valeur de l'île Saint- Barnabé}

Un chantier touristique : les attentes suscitées par un projet de mise en valeur

Afin de retenir les touristes de passage ${ }^{15}$, de prolonger le séjour des visiteurs et d'en attirer de nouveaux, Tourisme Rimouski a entrepris dans les années 2000 de diversifier son offre touristique en mettant en valeur l'histoire de l'île Saint-Barnabé et de ses habitants, en plus de son milieu naturel. Le projet consistait entre autres à aménager sur l'île des stations comprenant des panneaux d'interprétation, des objets et, dans certains cas, des chaises, des tables à pique-nique ou un abri. Les thèmes retenus comprenaient les naufrages autour de l'île, son rôle dans la contrebande d'alcool, les cultivateurs de l'île (dont ceux de la famille Lepage, étroitement associée aux origines de Rimouski) ${ }^{16}$ et surtout l'ermite Toussaint

15 Rimouski est sur la route de la Gaspésie, une destination touristique privilégiée des touristes québécois et étrangers. Dès la fin du XIXe siècle, la péninsule gaspésienne faisait partie de la Tourism Belt, un réseau de sites de villégiature des élites urbaines canadiennes et américaines ; avec l'ouverture de la route, le "tour" de la Gaspésie a offert l'une des premières expériences d'un tourisme populaire basé sur des circuits à parcourir en automobile. Voir GAGNON, Serge. L'échiquier touristique québécois. Sainte-Foy : Presses de l'Université du Québec, 2003, p. 119-125 et p. 264-273.

16 Installé dès la fin du XVIIe siècle, René Lepage de Sainte-Claire a été le premier seigneur résident de Rimouski. Son fils et son petit-fils, Pierre Lepage de Saint-Barnabé et Pierre Lepage de Saint-Barnabé II, ont signé en 1728 et en 1764 les documents qui autorisaient Toussaint Cartier à habiter l'île Saint-Barnabé. Un autre descendant du Seigneur, Louis-Jacques Lepage, 
Cartier. La station portant sur l'ermite devait être installée sur le site même où, selon la tradition orale, il aurait vécu.

Pour les responsables de cette mise en valeur, l'archéologie pouvait offrir des artefacts à exposer in situ, intégrés à la station sur Toussaint Cartier. On attendait donc de la culture matérielle qu'elle illustre et valide un discours établi d'avance, et non qu'elle l'alimente. Il est vrai que Rimouski elle-même avait été relativement peu exposée à l'archéologie et à la mise en valeur de la ressource archéologique, les rares travaux archéologiques ayant été menés dans des contextes préventifs et par des équipes de l'extérieur. L'opération la plus remarquable en archéologie historique, encore récente (20022003), avait été la fouille du premier cimetière de Rimouski (17121863), au centre-ville, à l'emplacement où devait être bâtie une salle de spectacle ; une vitrine dans le hall de cette dernière présente depuis quelques artefacts provenant des latrines du premier évêché (18701920), découvertes à cette occasion ${ }^{17}$.

Or, un nouveau créneau d'enseignement et de recherche en archéologie prenait alors forme à l'UQAR, à la faveur de l'embauche récente d'une professeure de géographie et d'un professeur d'histoire qui sont aussi archéologues. En 2008, à l'occasion de rencontres publiques sur l'avenir de l'île, une des archéologues de l'UQAR a établi un dialogue avec des employés de la Ville et pu ainsi faire valoir l'intérêt d'intégrer l'archéologie plus tôt dans la démarche, afin que ses résultats puissent contribuer à une mise en valeur éclairée.

C'est dans ce contexte que le nouveau Laboratoire d'Archéologie et de Patrimoine (LAP) de l'UQAR a été invité à

agriculteur, pêcheur et marinier, s'est établi sur l'île Saint-Barnabé avec sa famille dans la deuxième moitié du XIXe siècle et il y a vécu jusqu'à sa mort en 1903. La maison familiale a ensuite été laissée à l'abandon, servant de refuge aux chasseurs et aux excursionnistes jusqu'à sa destruction par un incendie accidentel en 1942; un panneau marque aujourd'hui son emplacement. Théodore Lepage, petit-fils de Louis-Jacques, venait d'acquérir les lots Lepage et s'y construisit un chalet; il cultiva les terres de son grand-père jusqu'en 1973, quand il mit fin à ses activités sur l'île pour s'établir à Rimouski (CHASSÉ, op. cit., p. 52-53). Il continua cependant à fréquenter son chalet sur l'île.

17 L'archéologue Gilles Rousseau et l'anthropologue Robert Larocque ont présenté leurs résultats à la population lors d'une conférence publique livrée dans la nouvelle salle de spectacle. Cet événement a connu un grand succès. Voir aussi ROUSSEAU, Gilles. Le cœur religieux de Rimouski : les interventions archéologiques de 2002 et 2003. In : Jean-René THUOT, Kurt VIGNOLA et Nicolas BEAUDRY (eds.). La cathédrale de Rimouski. Parcours, mémoires, récits. Rimouski : Les Éditions de l'Estuaire, 2017, p. 199-214. 
entreprendre des travaux archéologiques sur l'île Saint-Barnabé à l'été 2009. Tourisme Rimouski voyait d'un bon œil ce partenariat avec un organisme régional. La participation d'étudiants et les retombées éducatives étaient perçues comme des plus-values, même si cela pouvait se traduire par un rythme de travail plus lent que celui qu'aurait pu fournir une équipe professionnelle. Par ailleurs, comme les travaux devaient être menés durant la saison estivale alors que l'île est accessible aux visiteurs, Tourisme Rimouski souhaitait que l'équipe puisse les accueillir et échanger avec eux mais sans imposer de formule à cet égard. Quant au nouveau LAP, ce projet lui permettait de s'engager résolument dans la communauté locale et dans la mise en valeur de son patrimoine.

Un chantier universitaire : de l'archéologie préventive à la recherche appliquée

De 2009 à 2014, le LAP a mené six interventions archéologiques annuelles de quatre semaines chacune sur l'île Saint Barnabé. Cinq ont porté sur le site où Toussaint Cartier aurait vécu, la saison 2011 ayant été consacrée à la pointe est de l'île.

Les travaux de 2009 et 2010, menés en collaboration avec Ruralys $^{18}$, ont eu à la fois le caractère préventif d'une intervention préalable à un aménagement et le caractère académique d'une intervention menée dans le but d'acquérir des connaissances. Les questions demeuraient cependant déterminées par le projet de mise en valeur. Les représentants de la Ville souhaitaient notamment valider la localisation du site où, selon la tradition orale, aurait vécu Toussaint Cartier : sur sa rive sud de l'île, vers son centre, face à l'église et au noyau villageois. On espérait également préciser la forme de la maison de l'ermite, sur laquelle les témoignages de deuxième main du $\mathrm{XIX}^{\mathrm{e}}$ siècle se contredisent : elle est tantôt décrite comme une maison en colombage de 35 pieds sur 20 pieds avec un solage et une cheminée en pierre, tantôt comme une simple

18 Ruralys est un organisme patrimonial sans but lucratif basé à La Pocatière, dans la région du Bas-Saint-Laurent. 
«maisonnette » d'une pièce ${ }^{19}$; une gravure représentant les derniers moments de l'ermite, parue à la une du quotidien La Presse en 1906, la représente comme une cabane en bois rond ${ }^{20}$. Il a fallu cependant modérer les attentes : une intervention archéologique pouvait peutêtre confirmer une occupation du XVIII ${ }^{\mathrm{e}}$ siècle, contemporaine de l'ermite, mais il serait peu probable qu'elle puisse être associée avec certitude à Toussaint Cartier puisque d'autres ont pu fréquenter l'île de son vivant ou après lui.

Les sources historiques et la tradition orale promettaient déjà un excellent potentiel du site pour le XVIII ${ }^{\mathrm{e}}$ siècle, mais aussi pour les périodes antérieures et postérieures. En 1993, un inventaire archéologique s'intéressant à l'occupation amérindienne des îles du Saint-Laurent n'avait livré que des sondages négatifs à la pointe est de l'île ${ }^{21}$, mais celle-ci est battue par les vents alors que la rive sud de l'île est plus clémente; le site est en outre près de l'embouchure du seul cours d'eau de l'île. La période la plus prometteuse était le Sylvicole (3000 à $500 \mathrm{AA})$, la période la plus récente de la préhistoire : le site, à une altitude de 4 à $6 \mathrm{~m}$, appartient en effet à la paléo-terrasse marine de Mitis formée il entre 3000 et 1000 ans $\mathrm{AA}^{22}$. Après l'ermite, l'île a été habitée par des agriculteurs; elle a été fréquentée par des villégiateurs et des chasseurs; sa forêt a été exploitée au cours des $\mathrm{XIX}^{\mathrm{e}}$ et $\mathrm{XX}^{\mathrm{e}}$ siècles.

Dès les premiers jours de l'intervention de 2009, les travaux ont livré des artefacts du XVIII ${ }^{\mathrm{e}}$ siècle, mais aussi des artefacts autochtones du Sylvicole, ainsi que de riches assemblages du $\mathrm{XIX}^{\mathrm{e}}$ siècle et de la première moitié du $\mathrm{XX}^{\mathrm{e}}$. Ils ont aussi livré une assise de la fondation d'un bâtiment du XIX ${ }^{\mathrm{e}}$ siècle, qui semble avoir remployé l'emplacement et des matériaux d'une occupation du

19 La Charité, Claude. Toussaint Cartier, l'ermite de l'île Saint-Barnabé (I) : La maison du solitaire, petite cabane en bois rond ou grand ermitage ? Le Mouton Noir, n. 15(1), septembreoctobre 2009.

20 Gravure d'Albert-Samuel BRODEUR, La Presse, Montréal, 29 septembre 1906.

21 TREMBLAY, Roland, et VAILLANCOURT, J.-B. (1994). Rapport des activités archéologiques menées sur les îles du Bas-Saint-Laurent, Rapport déposé au ministère de la Culture du Québec, Montréal : Université de Montréal, Département d'anthropologie, 1993.

22 Dionne, Jean-Claude. État des connaissances sur la ligne de rivage Micmac de J. W. Goldthwait (estuaire du Saint-Laurent). Géographie physique et Quaternaire, n. 56(1), 2002, p. $97-121$. 
XVIII ${ }^{\mathrm{e}}$. Toussaint Cartier n'aurait donc été ni le premier habitant de l'île, ni le dernier occupant de ce site ${ }^{23}$.

Le site a été inscrit à l'Inventaire des sites archéologiques du Québec sous le code DcEd-9. Il a été perturbé par les occupations successives et par des labours, mais depuis 2009 il a été possible de circonscrire des zones présentant une plus forte densité d'artefacts du $\mathrm{XVIII}{ }^{\mathrm{e}}$ siècle. Les travaux ont également livré un niveau préservé du $\mathrm{XVIII}^{\mathrm{e}}$ siècle sous l'emprise du bâtiment, des dépotoirs et des vestiges d'autres bâtiments et aménagements des $\mathrm{XIX}^{\mathrm{e}}$ et $\mathrm{XX}^{\mathrm{e}}$ siècles, ainsi que des lambeaux d'un site préhistorique et un bel assemblage d'artefacts préhistoriques dont certains datent peut-être de l'Archaïque (6000-3000 AA).

\section{Un chantier étudiant : l'apprentissage de la pratique archéologique}

Le développement du LAP et de la recherche en archéologie à l'UQAR s'est accompagné du développement d'une offre de cours théoriques et pratiques, mais avant 2009, seul un petit nombre d'étudiants pouvait s'initier à la pratique archéologique outre-mer dans le cadre d'ententes bilatérales. L'ouverture du chantier de l'île Saint-Barnabé a permis d'offrir localement, et à un plus grand nombre, des formations pratiques en archéologie bien ancrées dans la pratique québécoise. Aux plus motivés, ce chantier a permis d'acquérir une expérience plus poussée et d'encadrer à leur tour des débutants.

En 2011, les travaux se sont déplacés à la pointe est de l'île Saint-Barnabé pour explorer des présumées caches de contrebande. Ils devaient contribuer à un projet de maitrise en histoire sur la patrimonialisation de la contrebande pendant les prohibitions canadienne (première moitié $\mathrm{du} \mathrm{XX}^{\mathrm{e}}$ siècle, dates différentes selon les provinces) et américaine (1919-1933). L'intervention devait notamment valider la fonction de fosses associées à la contrebande par la tradition orale, matérialisée par un panneau d'interprétation et une station de mise en valeur ${ }^{24}$; les travaux ont permis de dater une

23 SAVARD et al., loc. cit.

24 La station de mise en valeur présente un contenant de Hand Brand, un alcool qui était distribué dans le golfe et l'estuaire du Saint-Laurent à partir du territoire français de Saint- 
de ses fosses du premier tiers du $\mathrm{XX}^{\mathrm{e}}$ siècle, mais pas d'en confirmer la fonction.

En 2012, l'équipe du LAP est revenue sur le site DcEd-9, où aurait vécu l'ermite. À la faveur du projet PATER (patrimoine, enseignement, recherche), porté par l'UQAR et le Cégep de Rimouski, dont l'objectif était de favoriser la valorisation du patrimoine bas-laurentien dans l'enseignement et la recherche ${ }^{25}$, l'intervention archéologique a formellement pris la forme d'une École de fouilles archéologiques ${ }^{26}$ (figure 3 ), dont les trois premières éditions ont été offertes sur le site DcEd-9 de l'île Saint-Barnabé.

\section{Figure 3}

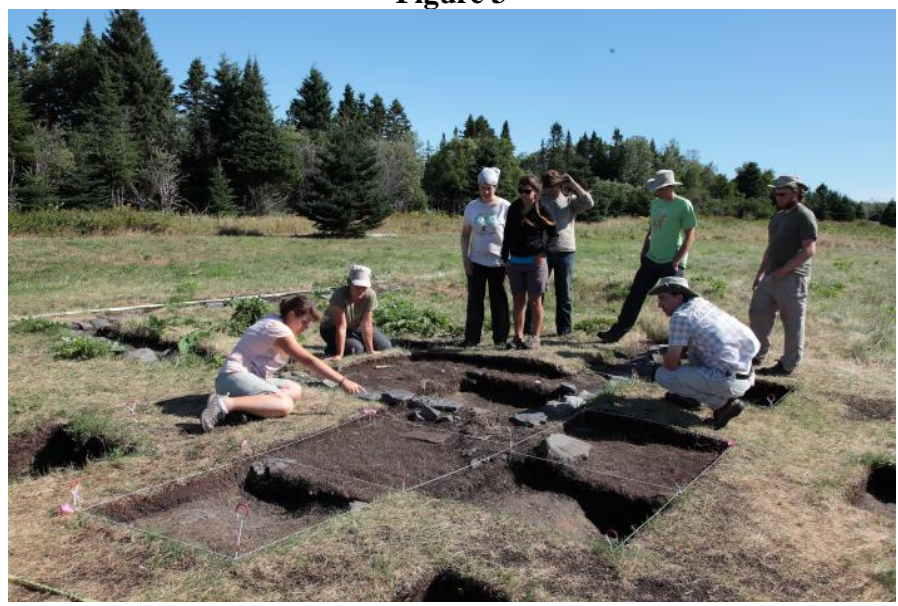

Un groupe d'étudiants de l'édition 2012 de l'école de fouilles (Nicolas Beaudry)

Pierre-et-Miquelon. Le contenant provient d'une fosse au Bic, en amont de Rimouski, mais sa présence sur l'île contribue à y matérialiser un récit de contrebande.

25 Le projet PATER a été financé par le ministère québécois de l'Éducation, du Loisir et du Sport de 2011 à 2014 ; voir Beaudry, Nicolas, Hébert, Karine, Marie, Guillaume, Pagniez, Renaud, Savard, Manon, Thuot, Jean-René, \& Vignola, Kurt. Patrimoine, enseignement et recherche (PATER): le patrimoine bas-laurentien au service de la formation collégiale et universitaire. In : Marie-Claude Larouche, Joanne Burgess, Joanne \& Nicolas Beaudry (éds.), Éveil et enracinement : approches pédagogiques innovantes du patrimoine culturel, Québec : Presses de l'Université du Québec, 2016, p. 229-246.

26 Savard, Manon, et Beaudry, Nicolas, Le patrimoine investi par l'archéologie. L'expérience du chantier-école de l'île Saint-Barnabé, dans LAROUCHE et al., op.cit., p. 171-187. 
Un chantier public : de la matérialisation du mythe à la performance archéologique

Les missions d'une université comprennent l'enseignement, la recherche et le service à la collectivité. Cette troisième est particulièrement importante pour une université régionale, qui agit comme un pôle scientifique et culturel, prend part au développement régional et contribue à la vitalité et au rayonnement du milieu. En tant que nouvel acteur régional, le LAP se devait de développer une pratique de l'archéologie adaptée aux réalités de l'Est du Québec et bien ancrée dans le milieu. Cela s'est manifesté notamment par le développement d'axes de recherche et d'intervention en archéologie environnementale, en archéologie des passés récents, ainsi qu'en archéologie publique, un champ de pratique et de recherche qui s'intéresse aux dimensions publiques de la pratique archéologique ainsi qu'aux interactions entre les archéologues et le public ${ }^{27}$.

Les travaux sur l'île Saint-Barnabé ont donné lieu à un effort de communication soutenu, de la part de Tourisme Rimouski comme de la part du LAP. Les médias locaux et le public local ont manifesté un grand intérêt pour les travaux avant même qu'ils ne commencent, en grande partie parce qu'ils devaient se dérouler sur le site où aurait vécu un personnage mythique cher aux Rimouskois ${ }^{28}$. Les travaux ont bénéficié chaque été d'une importante couverture médiatique à l'échelle locale et nationale sous la forme d'entrevues, de reportages et, à l'occasion de la création de l'École de fouilles en 2012, d'un documentaire $^{29}$. Des résultats ont été partagés avec le public par

27 MCDAVID, Carol. Bibliography of public archaeology sources, Archaeologies: Journal of the world Archaeological Congress, n. 7 (3), 2011, p. 657-666. Cette définition rend compte de deux approches de l'archéologie publique, en premier lieu celle du chercheur-acteur qui vise, dans ses interactions avec divers publics, la démocratisation de la ressource ou du savoir archéologique. Richardson, Lorna-Jane, et Almansa-Sánchez, Jaime. Do you even know what public archaeology is? Trends, theory, practice, ethics. World Archaeology, n. 47 (2), 2015, p. 194211; Schadla-Hall, Tim. Public archaeology. European Journal of Archaeology, n. 2 (2), 1999, p. 147-158. La seconde approche est celle du chercheur-observateur, qui s'intéresse à la production, à la consommation et aux représentations de l'archéologie. Voir Moshenska, Gabriel, What is public archaeology? Present Pasts, n. 1, 2009, p. 46-48. Ces deux positions ne s'excluent pas mutuellement et le LAP se réclame de l'une comme de l'autre.

28 SAVARD et al., loc. cit.

29 Voir par exemple CHAGNON, Sophie I., L'archéologie sur lị̂le Saint-Barnabé, Canal Savoir, Campus, émission 41, 12 février 2013, https://www.youtube.com/watch?v=gYzRkPv12LA. 
l'intermédiaire des médias, à l'occasion de conférences publiques, de publications ${ }^{30}$ et, à partir de 2013, par la mise en vitrine de quelques artefacts et l'affichage d'un panneau explicatif dans un petit bâtiment situé sur l'île, l'Espace Jaco-Lepage ${ }^{31}$.

L'île Saint-Barnabé étant ouverte aux visiteurs, c'est le site lui-même qui a été le principal lieu de rencontre entre les archéologues et le public (figures 4 et 5). Un questionnaire proposé aux visiteurs a permis de mieux connaître ce public et d'évaluer la contribution de l'activité archéologique à la fréquentation de l'île. Bon an, mal an, environ le tiers des personnes qui ont débarqué sur l'île pendant la durée des travaux s'est arrêté sur le site, soit environ 275 visiteurs sur le site en 2009, 575 en 2010 et environ 600 par saison par la suite (la fréquentation de l'île peut varier considérablement d'une saison à l'autre en fonction des conditions météorologiques et maritimes). Plus de la moitié provenaient de Rimouski ou des environs; les autres étaient des touristes provenant majoritairement des régions de Montréal et de Québec. La majorité des visiteurs visitaient l'île pour la première fois ${ }^{32}$. Les médias locaux et Tourisme Rimouski ont beaucoup contribué au succès du chantier auprès du public régional et des touristes, puisque plus des trois quarts des visiteurs sondés sur le site ont déclaré être au courant des travaux archéologiques et ont nommé l'archéologie parmi les motifs de leur visite; l'inscription du chantier au Mois de l'archéologie a aussi contribué à la visibilité des travaux à l'extérieur du Bas-SaintLaurent et a attiré un public plus spécialisé. Le site a en outre reçu chaque été la visite de représentants des médias et de groupes d'enfants.

30 Savard et al., loc. cit.

31 L'Espace Jaco-Lepage a été inauguré en 2013 sur le site de la grange de la famille Lepage, démolie l'année précédente pour des raisons de sécurité. Il présente aussi des outils agricoles et divers objets récupérés de la grange; un panneau propose des versions différentes du personnage de Toussaint Cartier.

32 SAVARD et BEAUDRY, loc. cit. 
Figure 4

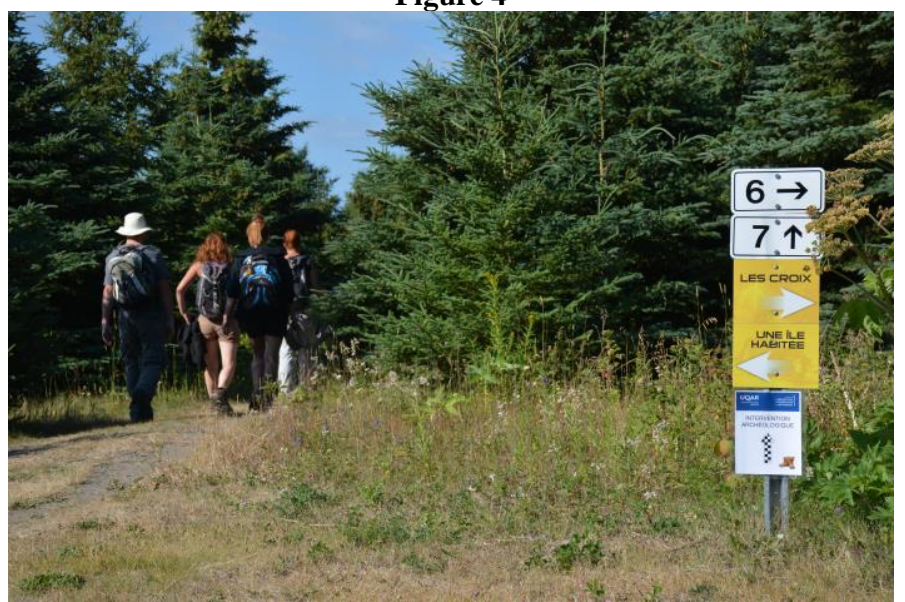

Signalisation à l'été 2014 (Nicolas Beaudry)

Figure 5

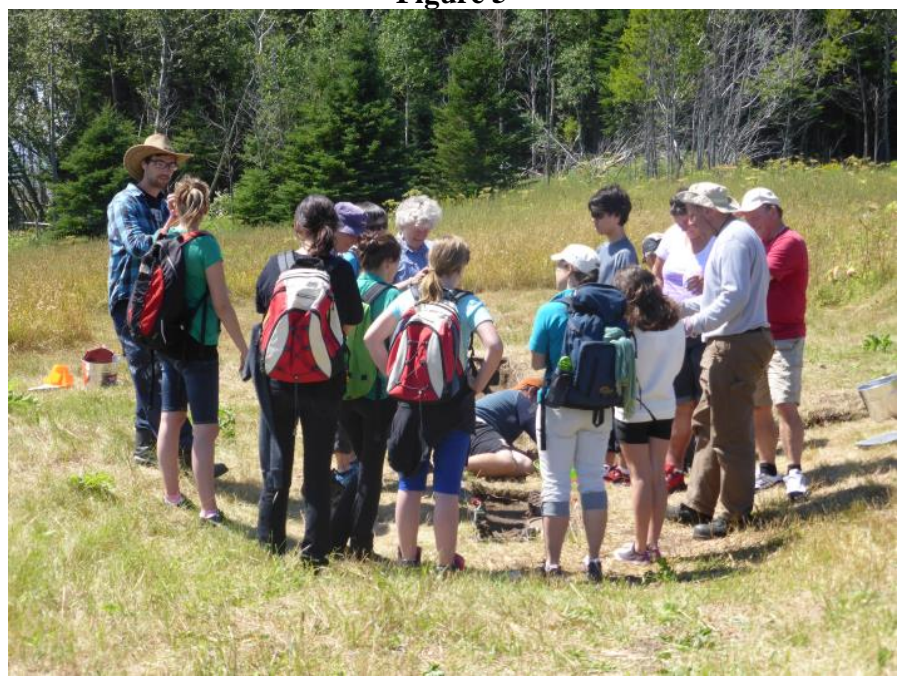

Groupe de visiteurs lors de l'édition 2013 de l'école de fouilles (Manon Savard) 


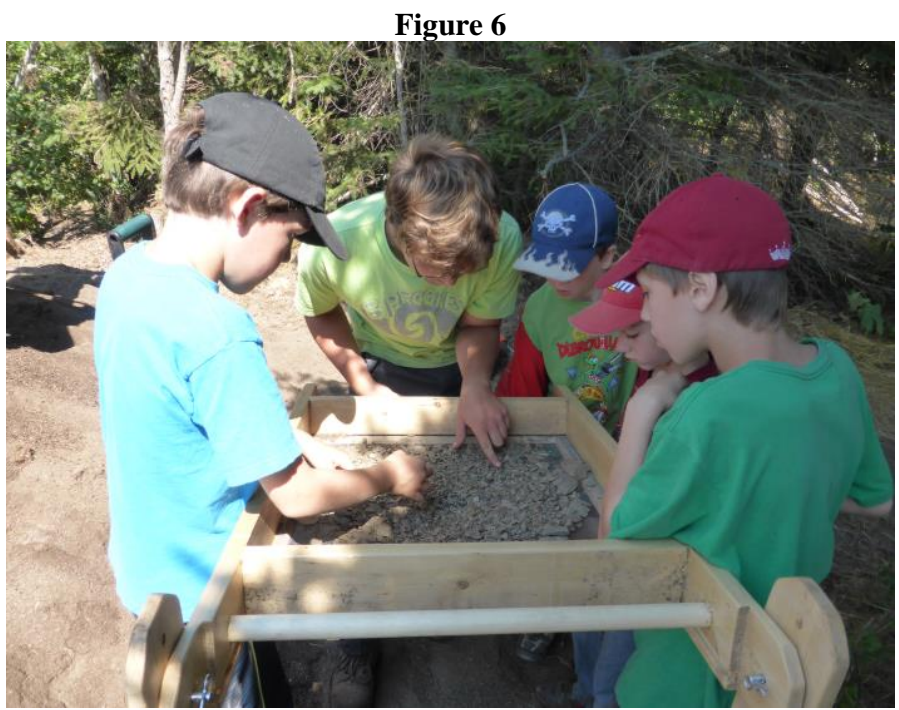

Groupe du club des Petits Débrouillards en visite sur l'île en 2013 (Manon Savard)

En 2009 et 2010, les attentes des visiteurs locaux étaient essentiellement les mêmes que celles de Tourisme Rimouski et des médias locaux: la plupart cherchaient à matérialiser l'histoire de Toussaint Cartier, à tel point que la visite sur l'île prenait pour certains le caractère d'un pèlerinage. En plus des questions auxquelles sont habituées les archéologues («Que cherchez-vous ? Pourquoi cherchez-vous ici?»), ces visiteurs posaient des questions sur l'ermite Toussaint Cartier sans nécessairement sentir le besoin de le nommer («Est-ce qu'il a vraiment existé? Avez-vous trouvé sa maison ?»). En mai 2011, la brasserie artisanale rimouskoise Le Bien, le Malt et Tourisme Rimouski lançaient conjointement la bière Toussaint-Cartier et un verre à l'effigie de l'ermite, premier souvenir mis en vente par Tourisme Rimouski et premier produit dérivé des excursions à l'île Saint-Barnabé ${ }^{33}$. Entre-temps, cependant, les par un ermite. Le Soleil, Québec, 6 juin 2011, http://www.lapresse.ca/le-soleil/affaires/agro- 
résultats des travaux archéologiques avaient largement dépassé la seule figure de l'ermite. Les visiteurs, les médias et Tourisme Rimouski ont pu découvrir une histoire beaucoup plus riche et les intérêts des uns comme des autres se sont étendus à d'autres phases d'occupation du site, de la préhistoire jusqu'au $\mathrm{XX}^{\mathrm{e}}$ siècle, qui a livré les assemblages d'artefacts les plus riches et les plus variés. En se déplaçant à la pointe est de l'île en 2011, les archéologues se sont détachés du personnage de l'ermite; la thématique de la contrebande d'alcool, résolument ancrée dans le $\mathrm{XX}^{\mathrm{e}}$ siècle, a aussi contribué à élargir les perspectives du public et des médias face à l'archéologie. Réduite pour l'occasion, l'équipe comprenait pour la première fois un étudiant dont la tâche était d'accueillir les visiteurs sur le site, ce dont la directrice du chantier s'était chargée jusque-là.

Depuis 2012, l'interaction avec le public est pleinement intégrée dans la démarche pédagogique de l'École de fouilles archéologiques de l'UQAR. L'accueil des visiteurs et l'interprétation du site est désormais assurée par les étudiants qui, à tour de rôle, deviennent guide d'un jour. En outre, depuis l'été 2013, les étudiants animent une activité de simulation de fouille offerte aux enfants sur le site (figure 7). Pour répondre aux attentes d'un public très divers et les nuancer au besoin, les étudiants doivent connaitre l'histoire de l'île, les récits qui s'y déploient et les valeurs patrimoniales qui y sont associées, mais ils doivent aussi pouvoir les aborder de façon critique, confronter les contradictions des sources, apprécier les différences d'échelles entre l'histoire et l'archéologie. Ils doivent pouvoir présenter les objectifs et les méthodes de l'intervention archéologique et suivre les interprétations préliminaires et changeantes des vestiges mis au jour ${ }^{34}$.

alimentaire/201106/05/01-4406231-la-brasserie-artisanale-le-bien-le-malt-lance-une-biere-inspireepar-un-ermite.php . consulté le 3 avril 2017.

34 Savard et BEAUDRY, loc. cit. 
Figure 7

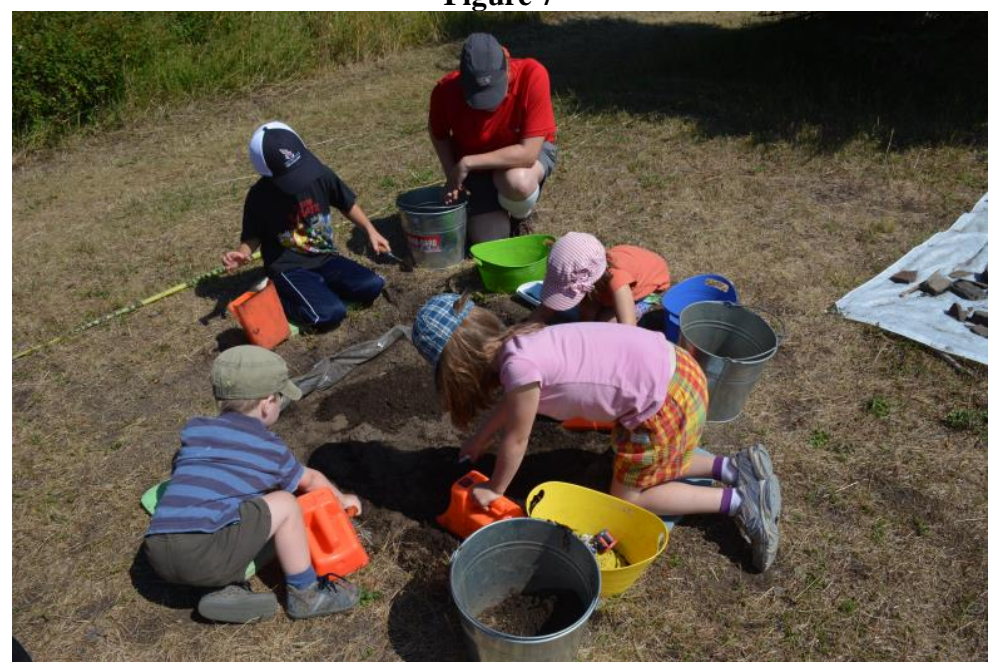

Activité de simulation de fouilles archéologiques en 2014 (Nicolas Beaudry)

Réciproquement, l'interaction avec les étudiants enrichit l'expérience des visiteurs. Les visites et la simulation de fouille permettent aux étudiants d'expliquer les processus de formation du site, la stratigraphie, les méthodes de la fouille, l'interprétation de la culture matérielle. Ils partagent leurs trouvailles, leur découverte de l'archéologie et leur patient apprentissage de sa pratique, à la première personne et dans leurs propres mots ${ }^{35}$. Leur regard et leur perspective ne sont pas ceux des professeurs ou des experts, mais ceux d'archéologues débutants, des néophytes dans lesquels les visiteurs peuvent aisément se projeter et dont ils peuvent partager l'émotion de la découverte. Les visiteurs sont d'autant plus à l'aise de leur proposer leur propre interprétation du site et des vestiges, particulièrement quand la fouille d'un dépotoir récent met au jour une culture matérielle qui leur est familière et leur permet de partager leur

35 Ce qui n'empêche pas d'exercer une certain vigilance sur la qualité de l'information qui est transmise aux visiteurs et, à plus forte raison, sur l'utilisation que certains seraient tentés d'en faire. Voir Richardson et Almansa-Sánchez, loc. cit., p 197-198. 
propre expérience avec les fouilleurs. Les dimensions pédagogique et publique du projet s'enrichissent ainsi mutuellement: alors que les contraintes imposées par la législation et la règlementation limitent la participation du public aux fouilles elles-mêmes, ces échanges entre les étudiants et les visiteurs contribuent à maintenir un dialogue ouvert entre les archéologues et la communauté et donne à celle-ci une voix dans la formation des étudiants comme dans l'interprétation du site ${ }^{36}$.

Au fil des saisons, les archéologues se sont ainsi ajoutés aux insulaires à la suite des cultivateurs, des pêcheurs, des contrebandiers, des forestiers ou des villégiateurs qui les ont précédés. L'île est désormais aussi celle des archéologues, comme en témoigne un roman-jeunesse publié en 2013 qui met en scène une école de fouilles archéologiques sur un site préhistorique de l'île Saint-Barnabé, sans la moindre mention d'un ermite ${ }^{37}$. Comme ceux des autres insulaires, les gestes et les pratiques des archéologues se prêtent désormais à l'interprétation et à la mise en valeur : l'intervention archéologique est devenue une performance offerte aux visiteurs par Tourisme Rimouski, qui a suspendu le projet d'une station de mise en valeur dédiée au seul personnage de l'ermite. On apprécie l'animation ordonnée du chantier, la virtuosité déployée dans la fouille et le nettoyage des tranchées, la richesse et la complexité des dépotoirs récents, le pouvoir d'évocation des vestiges et des artefacts in situ, la proximité avec les acteurs, le pittoresque du décor où s'étagent en arrière-plan la baie, la ville et les Hauts-Pays. En 2014, un calendrier promotionnel distribué par Tourisme Rimouski représentait l'île Saint-Barnabé en juin, quand débutent les excursions, par une photo du chantier-école dont rien ne laissait voir le caractère insulaire ${ }^{38}$.

L'École de fouille de l'UQAR a fait relâche en 2015 pour des raisons budgétaires ${ }^{39}$. Une activité de simulation de fouille a été

36 L’École de fouilles archéologiques s'est mérité en 2014 la Distinction Pascal-Parent de la Fondation de l'UQAR pour sa contribution à l'enseignement. En 2016, elle était lauréate des 10e Prix du patrimoine du Bas-Saint-Laurent, décernés par le Conseil de la culture du Bas-SaintLaurent, pour sa contribution à la diffusion et à l'interprétation du patrimoine régional.

37 Roberge, Sylvie. Le voleur d'artefacts. Lanoraie : Les Éditions de l'Apothéose, 2013.

38 L'École de fouilles se tenait alors en juillet et août.

39 Les activités de formation pratique du LAP se sont déplacées en 2015 sous la cathédrale de Rimouski et dans la vallée de la Matapédia et elles n'ont concerné qu'un petit nombre 
offerte pendant le Mois de l'archéologie à l'extérieur de l'Espace Jaco-Lepage, où sont exposés quelques artefacts, mais elle n'a pas été aussi fréquentée que les chantiers des années précédentes. Elle n'a pas été offerte sur l'île l'année suivante, alors que l'École de fouilles se tenait dans le parc national du Bic, en amont du centre de Rimouski, sur un autre site ouvert au public. L'équipe du LAP est retournée sur l'île en 2017, financée pour près de 50\% par la Ville de Rimouski qui souhaitait relancer l'animation archéologique sur l'île.

\section{La performance archéologique comme forme de mise en valeur}

Rétrospectivement, on constate que cette performance offerte par l'École de fouille résulte de la convergence d'évolutions parallèles (tableau 1) : les attentes de Tourisme Rimouski ont évolué au contact des archéologues et à la lumière de leurs résultats; un projet de recherche appliquée, puis de recherche fondamentale, est devenu une activité d'enseignement; l'offre touristique et les intérêts du public, d'abord centrés sur un personnage, se sont élargis aux différentes occupations du site puis à la pratique de l'archéologie. Si l'enseignement est en soi une performance, la diversification de l'offre d'animation proposée aux visiteurs et, notamment, l'ajout en 2013 d'une activité de simulations de fouilles archéologiques ont intégré les étudiants, responsables à tour de rôle de l'animation sur le site, et ceux-ci ont transmis aux visiteurs une partie de leurs apprentissages. Les étudiants sont donc devenus à leur tour les acteurs d'une performance pédagogique, ce qui a contribué à accroître la place de la pratique archéologique dans le discours offert aux

d'étudiants. voir BEAUDRY, Nicolas, Une exploration archéologique de la cathédrale SaintGermain de Rimouski. In : THUOT et al., op. cit., p. 215-235, et SAVARD, Manon, et BEAUDRY, Nicolas. Reconnaissance et prospection archéologiques dans la MRC de la Matapédia, août 2015, Rimouski : UQAR, LAP, 2016. Dans les deux cas, le LAP a cherché à entretenir un dialogue avec le public bien que les sites eux-mêmes n'aient pas été accessibles. 
visiteurs. L'attention accordée sur le site aux vestiges récents a aussi pu donner un relief particulier à la performance archéologique : en effet, l'archéologie du passé récent est volontiers associée à des pratiques artistiques qui exploitent un matériau très évocateur pour explorer des émotions esthétiques et susciter des réflexions relatives, en particulier, à la matérialité du monde contemporain et au passage du temps ${ }^{40}$.

\section{Tableau 1}

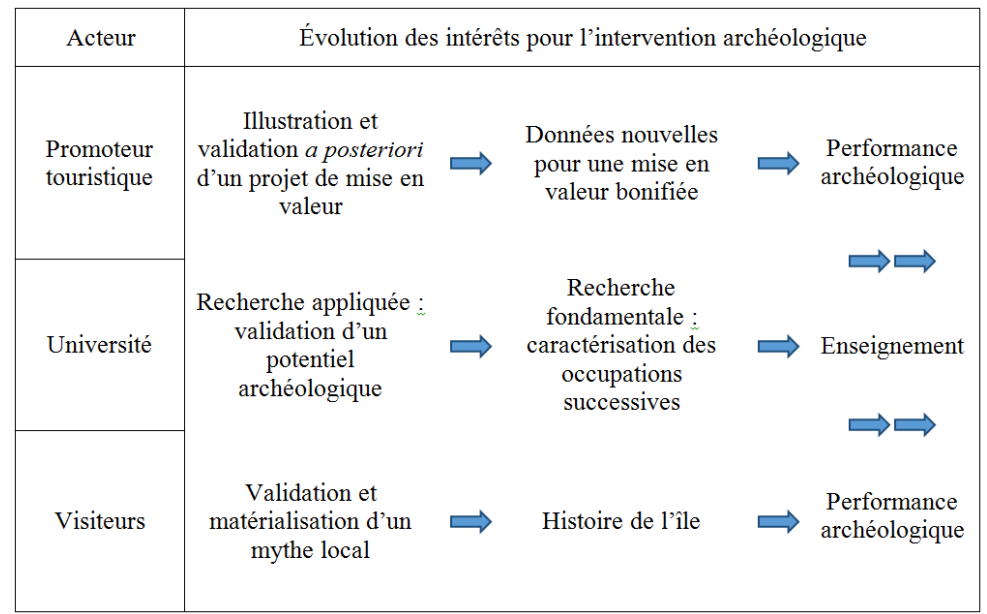

Évolution des intérêts des acteurs impliqués dans les interventions archéologiques sur l'île Saint-Barnabé et de l'interaction entre la performance archéologique, la recherche et l'enseignement.

40 Voir notamment HARRISSON, Rodney, et SCHOFIELD, John. After modernity: archaeological approaches to the contemporary pas. Oxford : Oxford University Press, 2010; OLSEN, Bjørnar, et PÉTURSDÓTTIRr, Póra (éds.). Ruin memories: materialities, aesthetics and the archaeology of the recent past. Londres : Routlegde, 2014. 


\section{Discussion}

Le vide apparent laissé sur l'île par l'absence de l'École de fouilles en 2015 et 2016 invite à une réflexion sur la performance archéologique comme forme de mise en valeur. Cette performance est un produit de consommation ${ }^{41}$ que l'on pourrait classer parmi les « expériences archéologiques », telles définies par Moshenska ${ }^{42}$. C'est cette «expérience archéologique » dont Tourisme Rimouski a fait la promotion auprès des visiteurs, en même temps que celle des expériences de randonnée et de camping offertes par l'environnement naturel de l'île ${ }^{43}$. L'expérience de 2016 au Parc national du Bic démontre que la performance de l'École de fouilles archéologiques peut être transposée sur d'autres sites accessibles au public. Pour être «authentique» aux yeux du touriste comme du visiteur local, cependant, une telle performance doit rester fermement ancrée dans l'histoire locale, dans ses lieux et dans ses personnages. Une performance qui ne serait fondée que sur la seule pratique archéologique n'aurait évidemment pas le pouvoir d'évocation du site, de son environnement, de sa culture matérielle, ni l'émotion et l'empathie avec le passé que suscitent la découverte. Ce serait faire de l'archéologie une fin en soi, réduite à un ensemble de méthodes et de savoirs techniques.

La première leçon qu'apprend l'apprenti-fouilleur est que la fouille archéologique est une opération destructrice, d'où l'importance de mettre en œuvre des méthodes de fouille et de documentation appropriées pour que l'archive matérielle détruite par la fouille donne lieu à une archive documentaire aussi complète et accessible que possible. On se contentera même souvent de ne fouiller qu'une partie d'un site pour préserver au moins une partie de l'archive matérielle, d'autant plus que la fouille génère des assemblages d'artefacts et d'écofacts et des obligations envers ces

41 Holtorf, Cornelius. Archaeology is a brand: the meaning of archaeology in contemporary popular culture. Walnut Creek, California : Left Coast Press, 2007.

42 Moshenska, op. cit.

43 Le rayonnement de cette «expérience » a assuré à l'École de fouilles une place privilégiée dans la promotion par l'UQAR de son offre de formation en sciences humaines. 
collections qui doivent être inventoriées et analysées, conservées, entreposées et rendues accessibles aux chercheurs.

Comme les minerais, les ressources archéologiques ne sont pas renouvelables; d'où aussi l'importance de justifier l'intervention archéologique si le site n'est pas menacé. Si l'acquisition de connaissances peut être un motif valable, la performance de la fouille peut-elle, à elle seule, justifier une activité archéologique?

Pour le moment, le site DcEd-9 continue de susciter plusieurs questions de recherche: le plan, la date, la nature et la fonction des bâtiments; la succession des occupations et des fonctions du site et le remploi des matériaux; la relation entre les occupations préhistoriques et les paléo-environnements, en fonction notamment de la fluctuation locale des niveaux marins. L'équipe du LAP est disposée à offrir une performance archéologique tout en poursuivant son enquête sur ces questions. Cependant, une telle performance archéologique sur l'île Saint-Barnabé ne pourra être pérenne et malgré son succès, une transition vers une autre forme de mise en valeur devra être prévue pour le site.

RECEBIDO EM: 01/06/2018

APROVADO EM: 12/07/2018 